\title{
A FIXED POINTS APPROACH TO STABILITY OF THE PEXIDER EQUATION
}

\author{
E. ELQORACHI, JOHN M. RASSIAS AND B. BOUIKHALENE
}

\begin{abstract}
Using the fixed point theorem we establish the Hyers-Ulam-Rassias stability of the generalized Pexider functional equation

$$
\frac{1}{|K|} \sum_{k \in K} f(x+k \cdot y)=g(x)+h(y), \quad x, y \in E
$$

from a normed space $E$ into a complete $\beta$-normed space $F$, where $K$ is a finite abelian subgroup of the automorphism group of the group $(E,+)$.
\end{abstract}

\section{Introduction and Preliminaries}

Under what condition does there exist a group homomorphism near an approximate group homomorphism? This question concerning the stability of group homomorphisms was posed by S. M. Ulam [59. In 1941, the Ulam's problem for the case of approximately additive mappings was solved by D. H. Hyers 21] on Banach spaces. In 1950 T. Aoki 4 provided a generalization of the Hyers' theorem for additive mappings and in 1978 Th. M. Rassias [48, generalized the Hyers' theorem for linear mappings by considering an unbounded Cauchy difference. The result of Rassias' theorem has been generalized by J.M. Rassias [45] and later by Găvruta [18 who permitted the Cauchy difference to be bounded by a general control function. Since then, the stability problems for several functional equations have been extensively investigated (cf. [4],.., [14, [16, $, \ldots, 51,[53, \ldots,[55]$ and 60]).

Let $E$ be a real vector space and $F$ be a real Banach space. Let $K$ be a finite abelian subgroup of $A u t(E)$ (the automorphism group of the group $(E,+),|K|$ denotes the order of $K$. Writing the action of $k \in K$ on $x \in E$ as $k \cdot x$, we will say that $(f, g, h): E \rightarrow F$ is a solution of the generalized Pexider functional equation, if

$$
\frac{1}{|K|} \sum_{k \in K} f(x+k \cdot y)=g(x)+h(y), \quad x, y \in E
$$

The generalized quadratic functional equation

$$
\frac{1}{|K|} \sum_{k \in K} f(x+k \cdot y)=f(x)+f(y), \quad x, y \in E
$$

and the generalized Jensen functional equation

$$
\frac{1}{|K|} \sum_{k \in K} f(x+k \cdot y)=f(x), \quad x, y \in E
$$

are particulars cases of equation (1.1).

The functional equations (1.1), (1.2) and (1.3) appeared in several works by $\mathrm{H}$. Stetkær, see for example [56, [57] and [58. We refer also to the recent studies by 
Ł. Radosław [51] and [52].

If we set $K=\{I, \sigma\}$, were $I: E \longrightarrow E$ denotes the identity function and $\sigma$ denote an additive function of $E$, such that $\sigma(\sigma(x))=x$, for all $x \in E$ then equation (1.1) reduces to the Pexider functionals equations

$$
\begin{gathered}
f(x+y)+f(x+\sigma(y))=g(x)+h(y), x, y \in E, \\
f(x+y)=g(x)+h(y), x, y \in E, \quad(\sigma=I) \\
f(x+y)+f(x-y)=g(x)+h(y), x, y \in E, \quad(\sigma=-I)
\end{gathered}
$$

Y. H. Lee and K. W. Jung 34 obtained the Hyers-Ulam-Rassias of the Pexider functional equation (1.5). Jung 28 and Jung and Sahoo 31 investigated the HyersUlam-Rassias stability of equation (1.6). Belaid et al. have proved the Hyers-Ulam stability of equation (1.1) and the Hyers-Ulam-Rassias stability of the functional equations (1.2), (1.3), (see [1, [10, 11] and [35] ).

Recently, Radosław [51 obtained the Hyers-Ulam-Rassias stability of equation (1.1). In 2003 L. Cădariu and V. Radu [8] notice that a fixed point alternative method is very important for the solution of the Hyers-Ulam stability problem. Subsequently, this method was applied to investigate the Hyers-Ulam-Rassias stability for Jensen functional equation, as well as for the additive Cauchy functional equation 9 by considering a general control function $\varphi(x, y)$, with suitable properties, using such an elegant idea, several authors applied the method to investigate the stability of some functional equations, see for example [2, [3], [32, 36] and [44].

The fixed point method was used for the first time by J. A. Baker [5] who applied a variant of Banach's fixed point theorem to obtain the Hyers Ulam stability of a functional equation in a single variable. For more information we refer to the recent studies by K. Cieplinśki [12].

In this paper, we will apply the fixed point method as in 8 . to prove the HyersUlam-Rassias stability of the functional equations (1.1), for a large classe of functions from a vector space $E$ into complete $\beta$-normed space $F$.

Now, we recall one of fundamental results of fixed point theory.

Let $X$ be a set. A function $d: X \times X \rightarrow[0, \infty]$ is called a generalized metric on $X$ if $d$ satisfies the following:

(1) $d(x, y)=0$ if and only if $x=y$;

(2) $d(x, y)=d(y, x)$ for all $x, y \in X$;

(2) $d(x, z) \leq d(x, y)+d(y, z)$ for all $x, y, z \in X$.

Theorem 1.1. 15] Suppose we are given a complete generalized metric space $(X, d)$ and a strictly contractive mapping $J: X \rightarrow X$, white the Lipshitz constant $L<1$. If there exists a nonnegative integer $k$ such that $d\left(J^{k} x, J^{k+1} x\right)<\infty$ for some $x \in X$, then the following are true:

(1) the sequence $J^{n} x$ converges to a fixed point $x^{*}$ of $J$;

(2) $x^{*}$ is the unique fixed point of $J$ in the set $Y=\left\{y \in X: d\left(J^{k} x, y\right)<\infty\right\}$;

(3) $d\left(y, x^{*}\right) \leq \frac{1}{1-L} d(y, J y)$ for all $y \in Y$.

Throughout this paper, we fix a real number $\beta$ with $0<\beta \leq 1$ and let $\mathbb{K}$ denote either $\mathbb{R}$ or $\mathbb{C}$. Suppose $E$ is a vector space over $\mathbb{K}$. A function $\|\cdot\|_{\beta}: E \longrightarrow[0, \infty)$ 
is called a $\beta$-norm if and only if it satisfies

(1) $\|x\|_{\beta}=0$, if and only if $x=0$;

(2) $\|\lambda x\|_{\beta}=|\lambda|^{\beta}\|x\|_{\beta}$ for all $\lambda \in \mathbb{K}$ and all $x \in E$;

(3) $\|x+y\|_{\beta} \leq\|x\|_{\beta}+\|y\|_{\beta}$ for all $x, y \in E$.

\section{MAIN RESULTS}

In the following theorem, by using an idea of Cădariu and Radu [8, 9, we prove the Hyers-Ulam-Rassias stability of the generalized Pexider functional equation (1.1).

Theorem 2.1. Let $E$ be a vector space over $\mathbb{K}$ and let $F$ be a complete $\beta$-normed space over $\mathbb{K}$. Let $K$ be a finite abelian subgroup of the automorphism group of $(E,+)$. Let $f: E \longrightarrow F$ be a mapping for which there exists a function $\varphi: E \times F \rightarrow$ $[0, \infty)$ and a constant $L<1$, such that

$$
\left\|\frac{1}{|K|} \sum_{k \in K} f(x+k \cdot y)-g(x)-h(y)\right\|_{\beta} \leq \varphi(x, y)
$$

and

$$
\sum_{k \in K} \varphi(x+k \cdot x, y+k \cdot y) \leq(|2 K|)^{\beta} L \varphi(x, y)
$$

for all $x, y \in E$. Then, there exists a unique solution $q: E \longrightarrow F$ of the generalierd quadratic functional equation (1.2) and a unique solution $j: E \longrightarrow F$ of the generalized Jensen functional equation (1.3) such that

$$
\begin{gathered}
\frac{1}{|K|} \sum_{k \in K} j(k \cdot x)=0 \\
\|f(x)-q(x)-j(x)-g(0)-h(0)\|_{\beta} \leq \frac{2}{2^{\beta}} \frac{1}{1-L} \chi(x, x)+\frac{1}{2^{\beta}} \frac{1}{1-L} \psi(x, x), \\
\|g(x)-q(x)-j(x)-g(0)\|_{\beta} \leq \varphi(x, 0)+\frac{2}{2^{\beta}} \frac{1}{1-L} \chi(x, x)+\frac{1}{2^{\beta}} \frac{1}{1-L} \psi(x, x)
\end{gathered}
$$

and

$$
\|h(x)-q(x)-h(0)\|_{\beta} \leq \frac{1}{2^{\beta}} \frac{1}{1-L} \psi(x, x)+\varphi(0, x)
$$

for all $x \in E$, where

$$
\begin{aligned}
\chi(x, y) & =\frac{|K|}{|K|^{\beta}} \varphi(0, y)+\varphi(x, y)+\varphi(x, 0)+\varphi(0, y) \\
& +\frac{1}{|K|^{\beta}} \sum_{k \in K}[\varphi(k \cdot x, y)+\varphi(k \cdot x, 0)]
\end{aligned}
$$

and

$$
\psi(x, y)=\frac{|K|}{|K|^{\beta}} \varphi(0, y)+\frac{1}{|K|^{\beta}} \sum_{k \in K}[\varphi(k \cdot x, y)+\varphi(k \cdot x, 0)] .
$$


Proof. Letting $y=0$ in (2.1), to obtain

$$
\|f(x)-g(x)-h(0)\|_{\beta} \leq \varphi(x, 0)
$$

for all $x \in E$. By using (2.7), (2.1) and the triangle inequality, we get (2.8)

$$
\begin{aligned}
\| \frac{1}{|K|} \sum_{k \in K} f(x+k \cdot y) & -f(x)-(h(y)-h(0))\left\|_{\beta} \leq\right\| \frac{1}{|K|} \sum_{k \in K} f(x+k \cdot y)-g(x)-h(y) \|_{\beta} \\
+\|g(x)-f(x)+h(0)\|_{\beta} & \leq \varphi(x, y)+\varphi(x, 0)
\end{aligned}
$$

for all $x, y \in E$. Replacing $x$ by 0 in (2.1), we get

$$
\left\|\frac{1}{|K|} \sum_{k \in K} f(k \cdot y)-g(0)-h(y)\right\|_{\beta} \leq \varphi(0, y)
$$

for all $y \in E$. So inequalities (2.8), (2.9) and the triangle inequality implies that (2.10)

$$
\begin{gathered}
\left\|\frac{1}{|K|} \sum_{k \in K} f(x+k \cdot y)-f(x)-\frac{1}{|K|} \sum_{k \in K} f(k \cdot y)+g(0)+h(0)\right\|_{\beta} \leq \frac{1}{|K|} \sum_{k \in K} f(x+k \cdot y)-f(x)-(h(y)-h(0)) \|_{\beta} \\
+\left\|\frac{1}{|K|} \sum_{k \in K} f(k \cdot y)-h(y)-g(0)\right\|_{\beta} \leq \varphi(x, y)+\varphi(x, 0)+\varphi(0, y)
\end{gathered}
$$

for all $x, y \in E$. Now, let

$$
\phi(x)=\frac{1}{|K|} \sum_{k \in K} f(k \cdot x)
$$

for all $x \in E$. Then, $\phi$ satisfies

$$
\frac{1}{|K|} \sum_{k \in K} \phi(k \cdot x)=\phi(x)
$$

for all $x \in E$. Furthermore, in view of (2.10), (2.12) and the triangle inequality, we have

$$
\left\|\frac{1}{|K|} \sum_{k^{\prime} \in K} \phi\left(x+k^{\prime} \cdot y\right)-\phi(x)-\phi(y)+g(0)+h(0)\right\|_{\beta}
$$$$
=\left\|\frac{1}{|K|} \sum_{k^{\prime} \in K} \frac{1}{|K|} \sum_{k \in K} f\left(k \cdot x+k k^{\prime} \cdot y\right)-\frac{1}{|K|} \sum_{k \in K} f(k \cdot x)-\frac{1}{|K|^{2}} \sum_{k, k^{\prime} \in K} f\left(k k^{\prime} \cdot y\right)+g(0)+h(0)\right\|_{\beta}
$$$$
\leq \frac{1}{|K|^{\beta}} \sum_{k \in K}\left\|\frac{1}{|K|} \sum_{k^{\prime} \in K} f\left(k \cdot x+k^{\prime} \cdot y\right)-f(k \cdot x)-\frac{1}{|K|} \sum_{k^{\prime} \in K} f\left(k^{\prime} \cdot y\right)+g(0)+h(0)\right\|_{\beta}
$$

$$
\leq \frac{1}{|K|^{\beta}} \sum_{k \in K}[\varphi(k \cdot x, y)+\varphi(k \cdot x, 0)]+\frac{|K|}{|K|^{\beta}} \varphi(0, y)=\psi(x, y) .
$$

Since $K$ is an abelian subgroup, so by using (2.2), we get

$$
\sum_{k \in K} \psi(x+k \cdot x, y+k \cdot y) \leq(2|K|)^{\beta} L \psi(x, y)
$$

for all $x, y \in E$. Let us consider the set $X:=\{g: E \longrightarrow F\}$ and introduce the generalized metric on $X$ as follows:

$$
d(g, h)=\inf \left\{C \in[0, \infty]:\|g(x)-h(x)\|_{\beta} \leq C \psi(x, x), \forall x \in E\right\} .
$$


Let $f_{n}$ be a Cauchy sequence in $(X, d)$. According to the definition of the Cauchy sequence, for any given $\varepsilon>0$, there exists a positive integer $N$ such that

$$
d\left(f_{n}, f_{m}\right) \leq \varepsilon
$$

for all integer $m, n$ such that $m \geq N$ and $n \geq N$. That is, by considering the definition of the generalized metric $d$

$$
\left\|f_{m}(x)-f_{n}(x)\right\|_{\beta} \leq \varepsilon \psi(x, x)
$$

for all integer $m, n$ such that $m \geq N$ and $n \geq N$, which implies that $f_{n}(x)$ is a Cauchy sequence in $F$, for any fixed $x \in E$. Since $F$ is complete, $f_{n}(x)$ converges in $F$ for each $x$ in $E$. Hence, we can define a function $f: E \longrightarrow F$ by

$$
f(x)=\lim _{n \longrightarrow \infty} f_{n}(x) .
$$

As a similar proof to [35], we consider the linear operator $J: X \rightarrow X$ such that

$$
(J h)(x)=\frac{1}{2|K|} \sum_{k \in K} h(x+k \cdot x)
$$

for all $x \in E$. By induction, we can easily show that

$$
\left(J^{n} h\right)(x)=\frac{1}{(2|K|)^{n}} \sum_{k_{1}, \ldots, k_{n} \in K} h\left(x+\sum_{i_{j}<i_{j+1}, k_{i j} \in\left\{k_{1}, \ldots, k_{n}\right\}}\left(k_{i_{1}} \ldots k_{i_{p}}\right) \cdot x\right)
$$

for all integer $n$.

First, we assert that $J$ is strictly contractive on $X$. Given $g$ and $h$ in $X$, let $C \in[0, \infty)$ be an arbitrary constant with $d(g, h) \leq C$, that is,

$$
\|g(x)-h(x)\|_{\beta} \leq C \psi(x, x)
$$

for all $x \in E$. So, it follows from (2.19), (2.14) and (2.21) we get

$$
\begin{aligned}
\|(J g)(x)-(J h)(x)\|_{\beta} & =\left\|\frac{1}{2|K|} \sum_{k \in K} g(x+k \cdot x)-\frac{1}{2|K|} \sum_{k \in K} h(x+k \cdot x)\right\|_{\beta} \\
& =\frac{1}{(2|K|)^{\beta}}\left\|\sum_{k \in K} g(x+k \cdot x)-h(x+k \cdot x)\right\|_{\beta} \\
& \left.\leq \frac{1}{(2|K|)^{\beta}} \sum_{k \in K} \| g(x+k \cdot x)-h(x+k \cdot x)\right) \|_{\beta} \\
& \leq \frac{1}{(2|K|)^{\beta}} C \sum_{k \in K} \psi(x+k \cdot x, x+k \cdot x) \\
& \leq C L \psi(x, x)
\end{aligned}
$$

for all $x \in E$, that is, $d(J g, J h) \leq L C$. Hence, we conclude that

$$
d(J g, J h) \leq L d(g, h)
$$

for any $g, h \in X$. Now, we claim that

$$
d(J(\phi-g(0)-h(0), \phi-g(0)-h(0))<\infty .
$$

By letting $y=x$ in (2.13), we obtain

$$
\|(J(\phi-g(0)-h(0)))(x)-(\phi-g(0)-h(0))(x)\|_{\beta}=\frac{1}{2^{\beta}}\left\|\frac{1}{|K|} \sum_{k \in K} \phi(x+k \cdot x)-2 \phi(x)+g(0)+h(0)\right\|_{\beta} \leq \frac{1}{2^{\beta}} \psi(x, x)
$$


for all $x \in E$, that is

$$
d(J(\phi-g(0)-h(0)), \phi-g(0)-h(0)) \leq \frac{1}{2^{\beta}}<\infty
$$

From Theorem 1.1, there exists a fixed point of $J$ which is a function $q: E \rightarrow F$ such that $\lim _{n \longrightarrow \infty} d\left(J^{n}(\phi-g(0)-h(0)), q\right)=0$. Since $d\left(J^{n}(\phi-g(0)-h(0)), q\right) \rightarrow 0$ as $n \rightarrow \infty$, there exists a sequence $\left\{C_{n}\right\}$ such that $\lim _{n \rightarrow \infty} C_{n}=0$ and $d\left(J^{n} \phi-\right.$ $g(0)-h(0), q) \leq C_{n}$ for every $n \in \mathbb{N}$. Hence, from the definition of $d$, we get

$$
\|\left(J ^ { n } \left(\phi-g(0)-h(0)(x)-q(x) \|_{\beta} \leq C_{n} \psi(x, x)\right.\right.
$$

for all $x \in E$. Therefore,

$$
\lim _{n \rightarrow \infty} \|\left(J^{n}(\phi-g(0)-h(0))(x)-q(x) \|_{\beta}=0,\right.
$$

for all $x \in E$.

Now, if we put $\kappa(x)=\phi(x)-g(0)-h(0)$, by using induction on $n$ we prove the validity of following inequality

$$
\left\|\frac{1}{|K|} \sum_{k \in K} J^{n} \kappa(x+k \cdot y)-J^{n} \kappa(x)-J^{n} \kappa(y)\right\|_{\beta} \leq L^{n} \psi(x, y) .
$$

In view of the commutativity of $K$ the inequalities (2.13) and (2.14) we have

$$
\begin{gathered}
\left\|\frac{1}{|K|} \sum_{k \in K} J f(x+k \cdot y)-J \kappa(x)-J \kappa(y)\right\|_{\beta} \\
=\left\|\frac{1}{|K|} \sum_{k \in K} \frac{1}{2|K|} \sum_{k_{1} \in K} \kappa\left(x+k \cdot y+k_{1} \cdot x+k_{1} k \cdot y\right)-\frac{1}{2|K|} \sum_{k_{1} \in K} \kappa\left(x+k_{1} \cdot x\right)-\frac{1}{2|K|} \sum_{k_{1} \in K} \kappa\left(y+k_{1} \cdot y\right)\right\|_{\beta} \\
\leq \frac{1}{\left(2|K|^{\beta}\right)} \sum_{k_{1} \in K}\left\|\frac{1}{|K|} \sum_{k \in K} \kappa\left(x+k_{1} \cdot x+k \cdot\left(y+k_{1} \cdot y\right)\right)-\kappa\left(x+k_{1} \cdot x\right)-\kappa\left(y+k_{1} \cdot y\right)\right\|_{\beta} \\
\leq \frac{1}{\left(2|K|^{\beta}\right)} \sum_{k_{1} \in K} \psi\left(x+k_{1} \cdot x, y+k_{1} \cdot y\right) \leq \frac{1}{(2|K|)^{\beta}}(2|K|)^{\beta} L \psi(x, y)=L \psi(x, y) .
\end{gathered}
$$

This proves (2.27) for $n=1$. Now, we assume that (2.27) is true for $n$. By using the commutativity of $K$, the inequalities (2.13), (2.14), we get

$$
\begin{gathered}
\left\|\frac{1}{|K|} \sum_{k \in K} J^{n+1} \kappa(x+k \cdot y)-J^{n+1} \kappa(x)-J^{n+1} \kappa(y)+g(0)+h(0)\right\|_{\beta} \\
=\| \frac{1}{|K|} \sum_{k \in K} \frac{1}{2|K|} \sum_{k^{\prime} \in K} J^{n} \kappa\left(x+k \cdot y+k^{\prime} \cdot x+k^{\prime} k \cdot y\right) \\
\quad-\frac{1}{2|K|} \sum_{k^{\prime} \in K} J^{n} \kappa\left(x+k^{\prime} \cdot x\right)-\frac{1}{2|K|} \sum_{k^{\prime} \in K} J^{n} \kappa\left(y+k^{\prime} \cdot y\right) \|_{\beta} \\
\leq \frac{1}{(2|K|)^{\beta}} \sum_{k^{\prime} \in K} \| \frac{1}{|K|} \sum_{k \in K} J^{n} \kappa\left(x+k^{\prime} \cdot x+k \cdot\left(y+k^{\prime} \cdot y\right)-J^{n} \kappa\left(x+k^{\prime} \cdot x\right)-J^{n} \kappa\left(y+k^{\prime} \cdot y\right) \|_{\beta}\right. \\
\leq \frac{1}{(2|K|)^{\beta}} \sum_{k^{\prime} \in K} L^{n} \psi\left(x+k^{\prime} \cdot x, y+k^{\prime} \cdot y\right) \leq L^{n+1} \psi(x, y),
\end{gathered}
$$


which proves (2.27) for $n+1$. Now, by letting $n \rightarrow \infty$, in (2.27), we obtain that $q$ is a solution of equation (1.2). According to the fixed point theorem (Theorem 1.1, (3)) and inequality (2.24), we get

$d(\phi-g(0)-h(0), q) \leq \frac{1}{1-L} d(J(\phi-g(0)-h(0)), \phi-g(0)-h(0)) \leq \frac{1}{2^{\beta}} \frac{1}{(1-L)}$

and so we have

$$
\| \phi(x)-q(x)-g(0)-h(0)) \| \leq \frac{1}{2^{\beta}} \frac{1}{(1-L)} \psi(x, x)
$$

for all $x \in E$. On the other hand if we put

$$
\omega(x)=f(x)-\phi(x)=f(x)-\frac{1}{|K|} \sum_{k \in K} f(k \cdot x)
$$

for all $x \in E$, it follows from inequalities (2.10), (2.13) and the triangle inequality that

$$
\begin{gathered}
\left\|\frac{1}{|K|} \sum_{k^{\prime} \in K} \omega\left(x+k^{\prime} \cdot y\right)-\omega(x)\right\|_{\beta} \\
=\left\|\frac{1}{|K|} \sum_{k^{\prime} \in K} f\left(x+k^{\prime} \cdot y\right)-\frac{1}{|K|} \sum_{k \in K} \phi(x+k \cdot y)-f(x)+\phi(x)\right\|_{\beta} \\
\leq\left\|-\frac{1}{|K|} \sum_{k \in K} \phi(x+k \cdot y)+\phi(x)+\phi(y)-g(0)-h(0)\right\|_{\beta} \\
+\left\|\frac{1}{|K|} \sum_{k^{\prime} \in K} f\left(x+k^{\prime} \cdot y\right)-f(x)-\frac{1}{|K|} \sum_{k^{\prime} \in K} f\left(k^{\prime} \cdot y\right)+g(0)+h(0)\right\|_{\beta} \\
\leq \frac{1}{|K|^{\beta}} \sum_{k \in K}[\varphi(k \cdot x, y)+\varphi(k \cdot x, 0)]+\frac{|K|}{|K|^{\beta}} \varphi(0, y)+\varphi(x, y)+\varphi(x, 0)+\varphi(0, y)=\chi(x, y)
\end{gathered}
$$
for all $x, y \in E$. By using the same definition for $X$ as in the above proof, the generalized metric on $X$

$$
d(g, h)=\inf \left\{C \in[0, \infty]:\|g(x)-h(x)\|_{\beta} \leq C \chi(x, x), \forall x \in E\right\} .
$$

and some ideas of [35], we will prove that there exists a unique solution $j$ of equation (1.3) such that

$$
\|\omega(x)-j(x)\|_{\beta} \leq \frac{1}{1-L} \chi(x, x)
$$

for all $x \in E$.

First, from (2.2) we can easily verify that $\chi(x, y)$ satisfies

$$
\sum_{k \in K} \chi(x+k \cdot x, y+k \cdot y) \leq(2|K|)^{\beta} L \chi(x, y)
$$

Let us consider the function $T: X \rightarrow X$ defined by

$$
(T h)(x)=\frac{1}{|2 K|} \sum_{k \in K} h(x+k \cdot x)
$$

for all $x \in E$. Given $g, h \in X$ and $C \in[0, \infty]$ such that $d(g, h) \leq C$, so we get

$$
\|(T g)(x)-(T h)(x)\|_{\beta}=\left\|\frac{1}{|2 K|} \sum_{k \in K} g(x+k \cdot x)-\frac{1}{|2 K|} \sum_{k \in K} h(x+k \cdot x)\right\|_{\beta}
$$




$$
\begin{gathered}
=\frac{1}{|2 K|^{\beta}}\left\|\sum_{k \in K}[g(x+k \cdot x)-h(x+k \cdot x)]\right\|_{\beta} \\
\leq \frac{1}{|2 K|^{\beta}} \sum_{k \in K}\|g(x+k \cdot x)-h(x+k \cdot x)\|_{\beta} \leq C L \chi(x, x)
\end{gathered}
$$

for all $x \in E$. Hence, we see that $d(T g, T h) \leq L d(g, h)$ for all $g, h \in X$. So $T$ is a strictly contractive operator.

Putting $y=x$ in (2.31), we have

$$
\left\|\frac{1}{|2 K|} \sum_{k \in K} \omega(x+k \cdot x)-\frac{1}{2} \omega(x)\right\|_{\beta} \leq \frac{1}{2^{\beta}} \chi(x, x)
$$

for all $x \in E$. So by triangle inequality, we get

$$
\left\|\frac{1}{|2 K|} \sum_{k \in K} \omega(x+k \cdot x)-\omega(x)\right\|_{\beta} \leq \frac{2}{2^{\beta}} \chi(x, x)
$$

for all $x \in E$, that is,

$$
d(T \omega, \omega) \leq \frac{2}{2^{\beta}} .
$$

From the fixed point theorem (Theorem 1.1), it follows that there exits a fixed point $j$ of $T$ in $X$ such that

$$
j(x)=\lim _{n \rightarrow \infty} \frac{1}{|2 K|^{n}} \sum_{k_{1}, \ldots, k_{n} \in K} \omega\left(x+\sum_{i_{j}<i_{j+1}, k_{i j} \in\left\{k_{1}, \ldots, k_{n}\right\}}\left[\left(k_{i_{1}}\right) \cdots\left(k_{i_{p}}\right)\right] \cdot x\right)
$$

for all $x \in E$ and

$$
d(\omega, j) \leq \frac{1}{1-L} d(T \omega, \omega) .
$$

So, it follows from (2.37) and (2.39) that

$$
\|\omega(x)-j(x)\|_{\beta} \leq \frac{2}{2^{\beta}} \frac{1}{1-L} \chi(x, x)
$$

for all $x \in E$.

By the same reasoning as in the above proof, one can show by induction that

$$
\left\|\frac{1}{|K|} \sum_{k \in K} T^{n} \omega(x+k \cdot y)-T^{n} \omega(x)\right\|_{\beta} \leq L^{n} \chi(x, y)
$$

for all $x, y \in E$ and for all $n \in \mathbb{N}$. Letting $n \rightarrow \infty$ in (2.41), we get that $j$ is a solution of the generalized Jensen functional equation (1.3).

From (2.11), (2.29) (2.30), (2.40) and the triangle inequality, we obtain

$$
\begin{aligned}
& \|f(x)-q(x)-j(x)-g(0)-h(0)\|_{\beta} \leq \frac{2}{2^{\beta}} \frac{1}{1-L} \chi(x, x)+\frac{1}{2^{\beta}} \frac{1}{1-L} \psi(x, x), \\
& \|g(x)-q(x)-j(x)-g(0)\|_{\beta} \leq \varphi(x, 0)+\frac{2}{2^{\beta}} \frac{1}{1-L} \chi(x, x)+\frac{1}{2^{\beta}} \frac{1}{1-L} \psi(x, x)
\end{aligned}
$$

and

$$
\|h(x)-q(x)-h(0)\|_{\beta} \leq \frac{1}{2^{\beta}} \frac{1}{1-L} \psi(x, x)+\varphi(0, x)
$$


for all $x \in E$.

Finally, in the following we will verify that the solution $j$ satisfies the condition

$$
\frac{1}{|K|} \sum_{k \in K} j(k \cdot x)=0
$$

for all $x \in E$ and we will prove the uniqueness of the solutions $q$ and $j$ which satisfy the inequalities (2.42) (2.43) and (2.44).

Due to definition of $\omega$, we get $\frac{1}{|K|} \sum_{k \in K} \omega(k \cdot x)=0$ for all $x \in E$, so we get $\frac{1}{|K|} \sum_{k \in K} T \omega(k \cdot x)=0, \frac{1}{|K|} \sum_{k \in K} T^{2} \omega(k \cdot x)=0, \ldots, \frac{1}{|K|} \sum_{k \in K} T^{n} \omega(k \cdot x)=0$. So, by letting $n \longrightarrow \infty$, we obtain the ralation (2.45).

Now, according to (2.44) and (2.2) we get by induction that

$$
\left\|J^{n}(h-h(0))(x)-q(x)\right\|_{\beta} \leq L^{n}\left[\frac{1}{2^{\beta}} \frac{1}{1-L} \psi(x, x)+\varphi(0, x)\right]
$$

for all $x \in E$ and for all $n \in \mathbb{N}$. So, by letting $n \longrightarrow \infty$, we get

$$
\lim _{n \longrightarrow \infty} J^{n}(h-h(0))(x)=q(x)
$$

for all $x \in E$, which proves the uniqueness of $q$.

In a similar way, by induction we obtain

$$
\left\|\Lambda^{n}(f-q-h(0)-g(0))(x)-j(x)\right\|_{\beta} \leq L^{n}\left[\frac{1}{1-L} \chi(x, x)+\frac{1}{2^{\beta}} \frac{1}{1-L} \psi(x, x)\right]
$$

for all $x \in E$ and for all $n \in \mathbb{N}$, where

$$
\Lambda l(x)=\frac{1}{|K|} \sum_{k \in K} l(x+k \cdot x) .
$$

Consequently, we have

$$
\lim _{n \longrightarrow \infty} \Lambda^{n}(f-q-h(0)-g(0))(x)=j(x)
$$

for all $x \in E$. This proves the uniqueness of the function $j$ and this completes the proof of theorem.

In the following, we will investigate some special cases of Theorem 2.1, with the new weaker conditions.

Corollary 2.2. Let $E$ be a vector space over $\mathbb{K}$. Let $K$ be a finite abelian subgroup of the automorphism group of $(E,+)$, Let $\alpha=\frac{\log (|K|)}{\log (2)}$. Fix a nonnegative real number $\beta$ such that $\frac{\alpha}{\alpha+1}<\beta<1$ and choose a number $p$ with $0<p<\beta+(\beta-1) \alpha$ and let $F$ be a complete $\beta$-normed space over $\mathbb{K}$. If a function $f: E \longrightarrow F$ satisfies

$$
\left\|\frac{1}{|K|} \sum_{k \in K} f(x+k \cdot y)-g(x)-h(y)\right\|_{\beta} \leq \theta\left(\|x\|^{p}+\|y\|^{p}\right)
$$

and $\|x+k \cdot x\| \leq 2\|x\|$, for all $k \in K$, for all $x, y \in E$ and for some $\theta>0$, then there exists a unique solution $q: E \longrightarrow F$ of the generalierd quadratic functional equation (1.2) and a unique solution $j: E \longrightarrow F$ of the generalized Jensen functional equation (1.3) such that

$$
\frac{1}{|K|} \sum_{k \in K} j(k \cdot x)=0
$$


$\|f(x)-q(x)-j(x)-g(0)-h(0)\|_{\beta} \leq \frac{\theta}{2^{\beta}} \frac{(2|K|)^{\beta}}{(2|K|)^{\beta}-2^{p}|K|}\left[\frac{|K|}{|K|^{\beta}}\left(6+6.3^{p}\right)+8\right]\|x\|^{p}$

$\|g(x)-q(x)-j(x)-g(0)\|_{\beta} \leq \frac{\theta}{2^{\beta}} \frac{(2|K|)^{\beta}}{(2|K|)^{\beta}-2^{p}|K|}\left[\frac{|K|}{|K|^{\beta}}\left(6+6.3^{p}\right)+8\right]\|x\|^{p}+\theta\|x\|^{p}$

and

$$
\|h(x)-q(x)-h(0)\|_{\beta} \leq \frac{\theta}{2^{\beta}} \frac{(2|K|)^{\beta}}{(2|K|)^{\beta}-2^{p}|K|}\left[\frac{|K|}{|K|^{\beta}}\left(2+2.3^{p}\right)\right]\|x\|^{p}+\theta\|x\|^{p}
$$

for all $x \in E$.

Proof. The proof follows from Theorem 2.1 by taking

$$
\varphi(x, y)=\theta\left(\|x\|^{p}+\|y\|^{p}\right)
$$

for all $x, y \in E$. Then we can choose $L=\frac{2^{p}}{2^{\beta}} \frac{|K|^{\beta}}{|K|}$ and we get the desired result.

Corollary 2.3. $(K=\{I\})$ Let $E$ be a vector space over $\mathbb{K}$. Fix a nonnegative real number $\beta$ less than 1 and choose a number $p$ with $0<p<1$ and let $F$ be a complete $\beta$-normed space over $\mathbb{K}$. If a function $(f, g, h): E \longrightarrow F$ satisfies

$$
\|f(x+y)-g(x)-h(y)\|_{\beta} \leq \theta\left(\|x\|^{p}+\|y\|^{p}\right)
$$

for all $x, y \in E$ and for some $\theta>0$, then there exists an unique additive function a: $E \longrightarrow F$ such that

$$
\begin{gathered}
\|f(x)-a(x)-g(0)-h(0)\|_{\beta} \leq \frac{\theta}{2^{\beta}} \frac{2^{\beta}}{2^{\beta}-2^{p}}\left[14+6.3^{p}\right]\|x\|^{p}, \\
\|g(x)-a(x)-g(0)\|_{\beta} \leq \frac{\theta}{2^{\beta}} \frac{2^{\beta}}{2^{\beta}-2^{p}}\left[14+6.3^{p}\right]\|x\|^{p}+\theta\|x\|^{p}
\end{gathered}
$$

and

$$
\|h(x)-a(x)-h(0)\|_{\beta} \leq \frac{\theta}{2^{\beta}} \frac{2^{\beta}}{2^{\beta}-2^{p}}\left[2+2.3^{p}\right]\|x\|^{p}+\theta\|x\|^{p}
$$

for all $x \in E$.

Corollary 2.4. $(K=\{I, \sigma\})$ Let $E$ be a vector space over $\mathbb{K}$. Let $K=\{I, \sigma\}$ where $\sigma$ is an volution of $E(\sigma(x+y)=\sigma(x)+\sigma(y)$ and $\sigma(\sigma(x))=x$ for all $x, y \in E)$. Fix a nonnegative real number $\beta$ such that $\frac{1}{2}<\beta<1$ and choose a number $p$ with $0<p<2 \beta-1$ and let $F$ be a complete $\beta$-normed space over $\mathbb{K}$. If a function $(f, g, h): E \longrightarrow F$ satisfies

$$
\|f(x+y)+f(x+\sigma(y))-g(x)-h(y)\|_{\beta} \leq \theta\left(\|x\|^{p}+\|y\|^{p}\right)
$$

and $\|x+\sigma(x)\| \leq 2\|x\|$, for all $x, y \in E$ and for some $\theta>0$, then there exists a unique solution $q: E \longrightarrow F$ of the generalierd quadratic functional equation

$$
f(x+y)+f(x+\sigma(y))=2 f(x)+2 f(y), \quad x, y \in E
$$

and a unique solution $j: E \longrightarrow F$ of the generalized Jensen functional equation

$$
f(x+y)+f(x+\sigma(y))=2 f(x), \quad x, y \in E
$$


such that

$$
j(\sigma(x))=-j(x)
$$

$\|f(x)-q(x)-j(x)-g(0)-h(0)\|_{\beta} \leq \frac{\theta}{2^{\beta}} \frac{4^{\beta}}{4^{\beta}-2^{p+1}}\left[\frac{2}{2^{\beta}}\left(6+6.3^{p}\right)+8\right]\|x\|^{p}$

$$
\|g(x)-q(x)-j(x)-g(0)\|_{\beta} \leq \frac{\theta}{2^{\beta}} \frac{4^{\beta}}{4^{\beta}-2^{p+1}}\left[\frac{2}{2^{\beta}}\left(6+6.3^{p}\right)+8\right]\|x\|^{p}+\theta\|x\|^{p}
$$

and

$$
\|h(x)-q(x)-h(0)\|_{\beta} \leq \frac{\theta}{2^{\beta}} \frac{4^{\beta}}{4^{\beta}-2^{p+1}}\left[\frac{2}{2^{\beta}}\left(2+2.3^{p}\right)\right]\|x\|^{p}+\theta\|x\|^{p}
$$

for all $x \in E$.

Corollary 2.5. Let $E$ be a vector space over $\mathbb{K}$ and let $F$ be a complete $\beta$-normed space over $\mathbb{K}$. Let $f: E \longrightarrow F$ be a mapping for which there exists a function $\varphi: E \times F \rightarrow[0, \infty)$ and a constant $L<1$, such that

$$
\|f(x+y)+f(x+\sigma(y))-g(x)-h(y)\|_{\beta} \leq \varphi(x, y)
$$

and

$$
\varphi(2 x, 2 y)+\varphi(x+\sigma(x), y+\sigma(y)) \leq 4^{\beta} L \varphi(x, y)
$$

for all $x, y \in E$. Then, there exists a unique solution $q: E \longrightarrow F$ of the generalierd quadratic functional equation (2.60) and a unique solution $j: E \longrightarrow F$ of the generalized Jensen functional equation (2.61) such that

$$
\begin{gathered}
j(\sigma(x))=-j(x) \\
\|f(x)-q(x)-j(x)-g(0)-h(0)\|_{\beta} \leq \frac{2}{2^{\beta}} \frac{1}{1-L} \chi(x, x)+\frac{1}{2^{\beta}} \frac{1}{1-L} \psi(x, x), \\
\|g(x)-q(x)-j(x)-g(0)\|_{\beta} \leq \varphi(x, 0)+\frac{2}{2^{\beta}} \frac{1}{1-L} \chi(x, x)+\frac{1}{2^{\beta}} \frac{1}{1-L} \psi(x, x)
\end{gathered}
$$

and

$$
\|h(x)-q(x)-h(0)\|_{\beta} \leq \frac{1}{2^{\beta}} \frac{1}{1-L} \psi(x, x)+\varphi(0, x)
$$

for all $x \in E$, where

$$
\begin{aligned}
& \chi(x, y)=\frac{2}{2^{\beta}} \varphi(0, y)+\varphi(x, y)+\varphi(x, 0)+\varphi(0, y) \\
& +\frac{1}{2^{\beta}}[\varphi(x, y)+\varphi(\sigma(x), y)+\varphi(x, 0)+\varphi(\sigma(x), 0)]
\end{aligned}
$$

and

$$
\psi(x, y)=\frac{2}{2^{\beta}} \varphi(0, y)+\frac{1}{2^{\beta}}[\varphi(x, y)+\varphi(\sigma(x), y)+\varphi(x, 0)+\varphi(\sigma(x), 0)]
$$


Corollary 2.6. Let $E$ be a vector space over $\mathbb{K}$ and let $F$ be a complete $\beta$-normed space over $\mathbb{K}$. Let $f: E \longrightarrow F$ be a mapping for which there exists a function $\varphi: E \times F \rightarrow[0, \infty)$ and a constant $L<1$, such that

$$
\|f(x+y)-g(x)-h(y)\|_{\beta} \leq \varphi(x, y)
$$

and

$$
\varphi(2 x, 2 y) \leq 2^{\beta} L \varphi(x, y)
$$

for all $x, y \in E$. Then, there exists an unique additive function $a: E \longrightarrow F$ such that

$$
\begin{gathered}
\|f(x)-a(x)-g(0)-h(0)\|_{\beta} \leq \frac{2}{2^{\beta}} \frac{1}{1-L} \chi(x, x)+\frac{1}{2^{\beta}} \frac{1}{1-L} \psi(x, x), \\
\|g(x)-a(x)-g(0)\|_{\beta} \leq \varphi(x, 0)+\frac{2}{2^{\beta}} \frac{1}{1-L} \chi(x, x)+\frac{1}{2^{\beta}} \frac{1}{1-L} \psi(x, x)
\end{gathered}
$$

and

$$
\|h(x)-a(x)-h(0)\|_{\beta} \leq \frac{1}{2^{\beta}} \frac{1}{1-L} \psi(x, x)+\varphi(0, x)
$$

for all $x \in E$, where

$$
\chi(x, y)=\varphi(0, y)+\varphi(x, y)+\varphi(x, 0)+\varphi(0, y)+\varphi(x, y)+\varphi(x, 0)
$$

and

$$
\psi(x, y)=\varphi(0, y)+\varphi(x, y)+\varphi(x, 0) .
$$

\section{REFERENCES}

[1] M. Ait Sibaha, B. Bouikhalene and E. Elqorachi, Hyers-Ulam-Rassias stability of the $K$ quadratic functional equation. J. Inequal. Pure and Appl. Math. 8 (2007), Article 89.

[2] M. Akkouchi, Stability of certain functional equations via a fixed point of Ćirić, Filomat $\mathbf{2 5}$ (2011), 121-127.

[3] M. Akkouchi, Hyers-Ulam-Rassias stability of Nonlinear Volterra integral equation via a fixed point approach, Acta Univ. Apulensis Math. Inform., 26 (2011), 257-266.

[4] T. Aoki, On the stability of the linear transformation in Banach spaces, J. Math. Soc. Japan, 2 (1950), 64-66.

[5] J. A. Baker, The stability of certain functional equations, Proc. Amer. Math. Soc., 112 (1991), 729-732.

[6] B. Bouikhalene, E. Elqorachi and Th. M. Rassias, On the Hyers-Ulam stability of approximately Pexider mappings. Math. Inequal. Appl., 11 (2008), 805-818.

[7] J. Brzdȩk, On a method of proving the Hyers-Ulam stability of functional equations on restricted domains, Austr. J. of Math. Anal. Appl., 6 (1), Article 4 (2009), 1-10.

[8] L. Cădariu and V. Radu, Fixed points and the stability of Jensens functional equation, Journal of Inequalities in Pure and Applied Mathematics, vol. 4, no. 1, (2003), article 4.

[9] L. Cădariu and V. Radu, On the stability of the Cauchy functional equation: a fixed point approach, Grazer Math. Berichte, vol. 346 (2004), 43-52.

[10] A. Charifi, B. Bouikhalene and E. Elqorachi, Hyers-Ulam-Rassias stability of a generalized Pexider functional equation, Banach J. Math. Anal., 1 (2007), 176-185.

[11] A. Charifi, B. Bouikhalene, E. Elqorachi and A. Redouani, Hyers-Ulam-Rassias Stability of a generalized Jensen functional equation, Australian J. Math. Anal. Appli. 19 (2009), 1-16.

[12] K. Cieplisński, Applications of fixed point theorems to the Hyers-Ulam stability of functional equations, A survey, Ann. Funct. Anal. 3 (2012), 151-164.

[13] Y. J. C. M. E. Gordji and S. Zolfaghari, Solutions and Stability of Generalized Mixed Type QC Functional Equations in Random Normed Spaces, Volume 2010, Article ID 403101, 16 pages. 
[14] H.G. Dales and M.S. Moslehian, Stability of mappings on multi-normed spaces, Glasgow Math. J. 49 (2007), 321-332.

[15] J. B. Diaz and B. Margolis, A fixed point theorem of the alternative, for contractions on a generalized complete metric space, Bulletin of the American Mathematical Society, vol. $\mathbf{7 4}$ (1968), 305-309.

[16] G. L. Forti, Hyers-Ulam stability of functional equations in several variables, Aequationes Math., 50 (1995), 143-190.

[17] G.-L. Forti and J. Sikorska, Variations on the Drygas equation and its stability, Nonlinear Anal.: Theory, Meth. Appl., 74 (2011), 343-350.

[18] P. Găvruta, A generalization of the Hyers-Ulam-Rassias stability of approximately additive mappings. J. Math. Anal. Appl., 184 (1994), 431-436.

[19] Z. Gajda, On stability of additive mappings, Internat. J. Math. Math. Sci., 14 (1991), 431434.

[20] M. E. Gordji, J. M. Rassias, M. B. Savadkouhi, Approximation of the Quadratic and Cubic Functional Equations in RN-spaces, EUROPEAN J. PURE, APPL. MATH., 2 (2009), 494507.

[21] D. H. Hyers, On the stability of the linear functional equation, Proc. Nat. Acad. Sci. U. S. A., 27 (1941), 222-224.

[22] D. H. Hyers, G. Isac and Th. M. Rassias, On the asymptoticity aspect of Hyers-Ulam stability of mappings, Proc. Amer. Math. Soc., 126 (1998), 425-430.

[23] D. H. Hyers, G. I. Isac and Th. M. Rassias, Stability of Functional Equations in Several Variables, Birkhäuser, Basel, 1998.

[24] D. H. Hyers and Th. M. Rassias, Approximate homomorphisms, Aequationes Math., 44 (1992), 125-153.

[25] M. Janfada and G; Sadeghi, Generalized Hyers-Ulam stability of a quadratic functional equation with involution in quasi- $\beta$-normed spaces, J. Appl. Math. Informatics, 29 (2011), 14211433.

[26] K.-W. Jun and Y.-H. Lee, A generalization of the Hyers-Ulam-Rassias stability of Jensen's equation, J. Math. Anal. Appl., 238 (1999), 305-315.

[27] S.-M. Jung, Hyers-Ulam-Rassias Stability of Functional Equations in Nonlinear Analysis, Springer, New York, 2011.

[28] S.-M. Jung, Stability of the quadratic equation of Pexider type, Abh. Math. Sem. Univ. Hamburg, 70 (2000), 175-190.

[29] S.-M. Jung, M.S. Moslehian and P K. Sahoo, Stability of generalized Jensen equation on restricted domains, J. Math Inequal., 4 (2010),191-206.

[30] S.-M. Jung and B. Kim, Local stability of the additive functional equation and its applications, IJMMS, (2003), 15-26.

[31] S.-M. Jung and P. K. Sahoo, Hyers-Ulam stability of the quadratic equation of Pexider type, J. Korean Math. Soc., 38 (3) (2001), 645-656.

[32] S.-M. Jung and Zoon-Hee Lee, A Fixed Point Approach to the Stability of Quadratic Functional Equation with Involution, Fixed Point Theory and Applications, V (2008), Article ID 732086, 11 pages.

[33] Pl. Kannappan, Functional Equations and Inequalities with Applications, Springer, New York, 2009.

[34] Y. H. Lee and K. W. Jung, A generalization of the Hyers-Ulam-Rassias stability of the Pexider equation, J. Math. Anal. Appl., 246 (2000), 627-638.

[35] Y. Manar, E. Elqorachi and B. Bouikhalene, Fixed point and Hyers-Ulam-Rassias stability of the quadratic and Jensen functional equations Nonlinear Funct. Anal. Appl., 15 (2) (2010), 273-284.

[36] M. S. Moslehian and A. Najati, Application of a fixed point theorem to a functional inequality, Fixed Point Theory 10 (2009), 141-149.

[37] M. S. Moslehian, The Jensen functional equation in non-Archimedean normed spaces, $J$. Funct. Spaces Appl., 7 (2009), 13-24.

[38] M. S. Moslehian and Gh. Sadeghi, Stability of linear mappings in quasi-Banach modules, Math. Inequal. Appl., 11 (2008), 549-557.

[39] A. Najati and M. B. Moghimi, Stability of a functional equation deriving from quadratic and additive functions in quasi-Banach spaces, J. Math. Anal. Appl., 337 (2008), 399-415. 
[40] A. Najati, On the stability of a quartic functional equation, J. Math. Anal. Appl., 340 (2008), 569-574.

[41] A. Najati and C. Park, Hyers-Ulam-Rassias stability of homomorphisms in quasi-Banach algebras associated to the Pexiderized Cauchy functional equation, J. Math. Anal. Appl., 335 (2007), 763-778.

[42] C. Park, On the stability of the linear mapping in Banach modules, J. Math. Anal. Appl., 275 (2002), 711-720.

[43] C. Park, Hyers-Ulam-Rassias stability of homomorphisms in quasi-Banach algebras, Bulletin Sci. Math., 132 (2008), 87-96.

[44] M. M. Pourpasha, J. M. Rassias, R. Saadati and S. M. Vaezpour, A fixed point approach to the stability of Pexider quadratic functional equation with involution, J. Ineq. Appl. (2010) Article ID 839639, doi:10.1155/2010/839639.

[45] J. M. Rassias, On approximation of approximately linear mappings by linear mappings, $J$. Funct. Anal., 46 (1982), 126-130.

[46] J. M. Rassias, Solution of a problem of Ulam, J. Approx. Theory., 57 (1989), 268-273.

[47] J. M. Rassias, On the Ulam stability of mixed type mappings on restricted domains, J. Math. Anal. Appl., 276 (2) (2002), 747-762.

[48] Th. M. Rassias, On the stability of linear mapping in Banach spaces, Proc. Amer. Math. Soc., 72 (1978), 297-300.

[49] Th. M. Rassias, The problem of S. M. Ulam for approximately multiplicative mappings, $J$. Math. Anal. Appl., 246 (2000), 352-378.

[50] Th. M. Rassias and J. Brzdęk, Functional Equations in Mathematical Analysis, Springer, New York, 2011.

[51] Radosław 1 . The solution and the stability of the Pexiderized $K$-quardratic functional equation, 12th Debrecen-Katowice Winter Seminar on Functional Equationsand Inequalities, Hajdúszoboszló, Hungary, January 25-28, 2012.

[52] Radosław ł. Some generalization of Cauchys and the quadratic functional equations, Aequationes Math., 83 (2012), 75-86.

[53] J. Schwaiger, The functional equation of homogeneity and its stability properties, Österreich. Akad. Wiss. Math.-Natur, Kl, Sitzungsber. Abt. II (1996), 205, 3-12.

[54] F. Skof, Approssimazione di funzioni $\delta$-quadratic su dominio restretto, Atti. Accad. Sci. Torino Cl. Sci. Fis. Mat. Natur., 118 (1984), 58-70.

[55] F. Skof, Sull'approssimazione delle applicazioni localmente $\delta$-additive, Atti. Accad. Sci. Torino Cl. Sci. Fis. Mat. Natur. 117 (1983), 377-389.

[56] H. Stetkær, Functional equations on abelian groups with involution. Aequationes Math. 54 (1997), 144-172.

[57] H. Stetkær, Operator-valued spherical functions, J. Funct. Anal., 224, (2005), 338-351.

[58] H. Stetkær, Functional equations and matrix-valued spherical functions. Aequationes Math. 69 (2005), 271-292.

[59] S. M. Ulam, A Collection of Mathematical Problems, Interscience Publ. New York, 1961. Problems in Modern Mathematics, Wiley, New York 1964.

[60] D. Yang, Remarks on the stability of Drygas equation and the Pexider-quadratic equation, Aequationes Math., 68 (2004), 108-116.

Elhoucien Elqorachi,

Department of Mathematics,

Faculty of Sciences, Ibn Zohr University, Agadir, Morocco,

E-mail: elqorachi@hotmail.com

John Michael Rassias,

National and Capodistrian University of Athens

Pedagogical Department E.E. Section of Mathematics and Informatics 4,

Agamemnonos Str., Aghia Paraskevi, Attikis 15342, Greece" .

E-mail: loannis.Rassias@primedu.uoa.gr; jrassias@primedu.uoa.gr; jrass@otenet.gr 
Bouikhalene Belaid

Department of Mathematics,

University Sultan Moulay Slimane, Faculty of Technical Sciences,

Béni-Mellal, Morocco

E-mail: bbouikhalene@yahoo.fr 